\title{
The role of platelet to lymphocyte ratio and neutrophil to lymphocyte ratio in ulcerative colitis
}

\author{
Yujin Jeong ${ }^{1}$, Seong Ran Jeon ${ }^{1}$, Hyun Gun Kim ${ }^{1}$, Jung Rock Moon ${ }^{1}$, Tae Hee Lee ${ }^{1}$, Jae Young Jang ${ }^{1}$, Jun-Hyung Cho ${ }^{1}$, \\ Jun Seok Park ${ }^{1}$, Heesu Park ${ }^{1}$, Ki-hun Lee ${ }^{1}$, Jin-Oh Kim ${ }^{1}$, Joon Seong Lee ${ }^{1}$, Bong Min Ko ${ }^{1}$, Suyeon Park ${ }^{2}$ \\ ${ }^{1}$ Digestive Disease Center, Institute for Digestive Research and ${ }^{2}$ Department of Biostatistics, Soonchunhyang University College of Medicine, \\ Seoul, Korea
}

Background/Aims: Neutrophil to lymphocyte ratio (NLR) and platelet to lymphocyte ratio (PLR) can serve as biomarkers for diagnosing and assessing disease activity in ulcerative colitis (UC). We investigated their clinical significance in UC. Methods: We analyzed 48 patients with UC who underwent measurement of fecal calprotectin (FC) and endoscopy and 96 age- and sexmatched healthy controls. NLR and PLR were compared between the patients and healthy controls. The endoscopic activity was divided into 2 groups: group 1 (mild to moderate inflammation) and group 2 (severe inflammation) according to the Mayo endoscopic subscore in UC. Results: To diagnose UC, the optimal cutoff of NLR and PLR was 2.26 (sensitivity 54.2\%; specificity 90.6\%; positive likelihood ratio 5.778, 95\% confidence interval [CI] 2.944-11.339; area under the curve [AUC] 0.774, 95\% CI, $0.690-0.859$ ) and 179.8 (sensitivity $35.4 \%$; specificity 90.6\%; positive likelihood ratio 3.778, 95\% CI 1.821-7.838; AUC 0.654, 95\% CI 0.556-0.753), respectively. The optimal cutoff to differentiate group 1 and group 2 was 3.44, 175.9, and $453 \mu \mathrm{g} / \mathrm{g}$ for NLR, PLR, and FC, respectively (sensitivity, $63.6 \%$ vs. $90.9 \%$ vs. $81.8 \%$; specificity, $81.1 \%$ vs. $78.4 \%$ vs. $73.0 \%$; positive likelihood ratio, 3.364 vs. 4.205 vs. 3.027 ; AUC, 0.714 vs. 0.897 vs. 0.813). PLR had the highest AUC and positive likelihood ratio. Conclusions: NLR and PLR help differentiate patients with UC from healthy controls. NLR, PLR, and FC indicate endoscopic activity and may reflect intestinal mucosal conditions. (Intest Res 2021;19:62-70)

Key Words: Colitis, ulcerative; Neutrophil to lymphocyte ratio; Platelet to lymphocyte ratio; Fecal calprotectin

\section{INTRODUCTION}

Ulcerative colitis (UC) causes idiopathic, chronic, and relapsing inflammation of the gastrointestinal (GI) tract. The incidence of UC has rapidly increased in Asia. ${ }^{1}$ Endoscopy plays an important role in the diagnosis, treatment, and monitoring of disease activity in UC. ${ }^{2}$ The new therapeutic goals for inflammatory bowel disease (IBD) are as follows-mucosal healing or the suppression of disease progression, decreased hospitalization time, decreased need for surgery and prolonged clini-

Received November 28, 2019. Revised March 16, 2020.

Accepted March 19, 2020.

Correspondence to Seong Ran Jeon, Digestive Disease Center, Institute

for Digestive Research, Soonchunhyang University College of Medicine, 59

Daesagwan-ro, Yongsan-gu, Seoul 04401, Korea. Tel: +82-2-709-9202, Fax:

+82-2-709-9581, E-mail:94jsr@hanmail.net cal remission. ${ }^{3}$ However, the assessment of mucosal activity via endoscopy is invasive, inconvenient, and costly, and may be inappropriate in patients with severe UC. Noninvasive inflammatory biomarkers of IBD, such as the white blood cell (WBC) count, erythrocyte sedimentation rate (ESR), and C-reactive protein (CRP) are used in clinical practice. However, no ideal biomarker has been identified to assess mucosal activity in IBD as the currently used biomarkers are nonspecific. ${ }^{4-6}$ Therefore, there is an unmet clinical need to identify biomarkers that can replace the need for an endoscopy to assess mucosal disease activity.

Fecal calprotectin (FC) is a marker of gut inflammation and shows a good correlation with endoscopic inflammation in UC. ${ }^{7}$ However, its correlation with clinical indices is weaker as compared with endoscopic findings. ${ }^{8-10}$ The neutrophil to lym- 
phocyte ratio (NLR) and platelet to lymphocyte ratio (PLR) and can be easily calculated from the complete blood count (CBC) and are simpler and less expensive biomarkers compared with FC. NLR and PLR can serve as useful biomarkers for diagnosing and predicting mucosal inflammation in UC., ${ }^{2,11}$

However, the clinical implications of NLR and PLR in patients with UC are inconsistent. Therefore, we investigated if NLR and PLR can help differentiate patients with UC from healthy controls. We evaluated the usefulness of NLR, PLR, and FC as disease activity biomarkers in UC.

\section{METHODS}

\section{Study Design and Subjects}

We retrospectively analyzed 48 patients with UC and 96 healthy controls who underwent colonoscopy and laboratory tests between January 2015 and October 2016 at the Soonchunhyang University Hospital (Fig. 1). The healthy controls included subjects who had undergone a health checkup at our hospital and had normal colonoscopy findings. Patients were matched to the healthy controls in a 1:2 ratio based on age and sex during the study period. Patients with UC were classified according to the Montreal classification. ${ }^{12}$ The exclusion criteria included previous bowel resection, indeterminate colitis, infection, neoplastic disorders, hematologic disease, heart or pulmonary disease, autoimmune disease, hepatosplenic disease, and renal insufficiency. The patient's age, sex, disease duration, medical and operation history, disease and endoscopic activity score, classification, laboratory findings, and disease treatment were recorded. This study was approved by the Institutional Review Board of our hospital (IRB No. SCHUH 2017-12-014-001). The informed consent was waived.

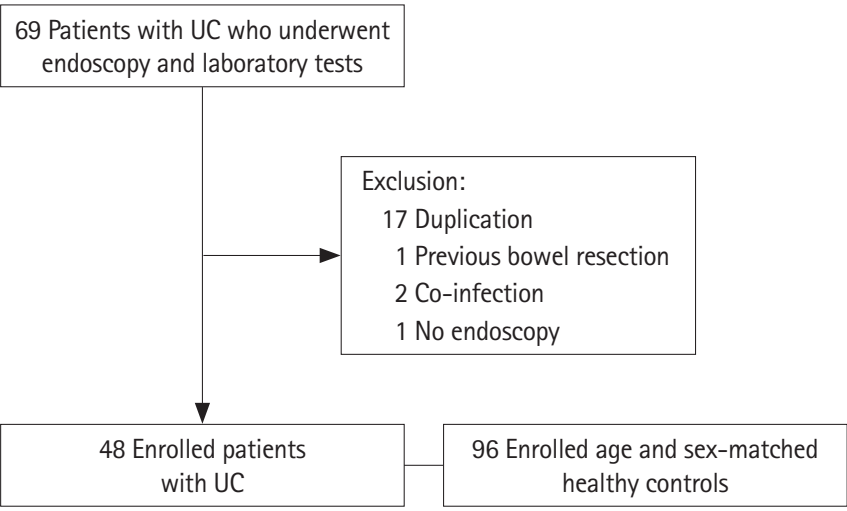

Fig. 1. Flowchart of the study subjects. UC, ulcerative colitis.

\section{Laboratory Values}

NLR and PLR were calculated by dividing the absolute neutrophil count by the absolute lymphocyte count and dividing the absolute platelet count by the absolute lymphocyte count, respectively. In healthy controls, the findings of the blood test on the day of the endoscopy were used. In patients with UC, findings of blood and FC tests performed within 1 month and of the endoscopy within 3 months were used. The medical time

Table 1. Demographic and Clinical Characteristics of the Study Population

\begin{tabular}{|c|c|}
\hline Variable & UC $(n=48)$ \\
\hline Age at UC diagnosis (yr) & $38.9 \pm 14.8$ \\
\hline Male sex & $26(54.2)$ \\
\hline Previous operation history & $5(10.4)$ \\
\hline Appendectomy & $1(20.0)$ \\
\hline Perianal operation & $2(40.0)$ \\
\hline Others $^{\mathrm{a}}$ & $2(40.0)$ \\
\hline \multicolumn{2}{|c|}{ Disease extension at diagnosis at diagnosis } \\
\hline E1 (proctitis) & $18(37.5)$ \\
\hline E2 (left-sided colitis) & $10(20.8)$ \\
\hline E3 (pan-colitis) & $20(41.7)$ \\
\hline \multicolumn{2}{|l|}{ Disease activity at diagnosis } \\
\hline Clinical remission (Mayo score 0 -2) & $1(2.1)$ \\
\hline Mild activity (Mayo score 3-5) & $17(35.4)$ \\
\hline Moderate activity (Mayo score 6-10) & $28(58.3)$ \\
\hline Severe activity (Mayo score 11-12) & $2(4.2)$ \\
\hline \multicolumn{2}{|c|}{ Disease activity at NLR, PLR and FC measurement } \\
\hline Clinical remission (Mayo score 0-2) & $9(18.8)$ \\
\hline Mild activity (Mayo score 3-5) & $19(39.6)$ \\
\hline Moderate activity (Mayo score 6-10) & $15(31.2)$ \\
\hline Severe activity (Mayo score 11-12) & $5(10.4)$ \\
\hline \multicolumn{2}{|c|}{ Medication use at NLR, PLR and FC measurement } \\
\hline 5 -ASA & $33(68.7)$ \\
\hline $5-A S A+A Z A$ & $5(10.4)$ \\
\hline 5-ASA+steroid & $4(8.3)$ \\
\hline $5-A S A+$ steroid+AZA & $2(4.2)$ \\
\hline $5-A S A+A Z A+a n t i-T N F$ & $2(4.2)$ \\
\hline 5-ASA+anti-TNF & $2(4.2)$ \\
\hline \multicolumn{2}{|c|}{$\begin{array}{l}\text { Values are presented as mean } \pm \text { standard deviation or number (\%). } \\
\text { a Others: hysterectomy, transurethral resection of bladder. } \\
\text { UC, ulcerative colitis; NLR, neutrophil to lymphocyte ratio; PLR, platel } \\
\text { to lymphocyte ratio; FC, fecal calprotectin; 5-ASA, 5-aminosalicylic aci } \\
\text { AZA, azathioprine; TNF, tumor necrosis factor. }\end{array}$} \\
\hline
\end{tabular}


interval between the laboratory tests and endoscopy was 2 days (interquartile range, 0-19 days) and the medical time interval between $\mathrm{FC}$ measurement and endoscopy was 4.5 days (interquartile range, 1-34 days). All stool samples were stored at $-20^{\circ} \mathrm{C}$, thawed, and analyzed by an enzyme-linked immunosorbent assay test (Ridascreen Calprotectin test; R-Biopharm, Darmstadt, Germany).

\section{Assessment of Clinical and Endoscopic Activities}

The UC disease activity was evaluated using the Mayo score. The endoscopic activity was assessed using the Mayo endoscopic subscore. Mayo endoscopic subscore grades were as follows-grade 0: normal or inactive, grade 1: mild, grade 2: moderate, and grade 3: severe. ${ }^{13}$ In pan-colitis or left-sided colitis, endoscopic activity was scored based on the most inflamed segment of the colon. The patients were divided into 2 groups based on the severity of the degree of inflammation (group 1: no, mild, and moderate inflammation vs. group 2: severe inflammation) to evaluate the usefulness of NLR, PLR, and FC as biomarkers of disease activity in UC. The primary endpoint was the ability of NLR and PLR to serve as biomarkers for UC and the ability of NLR, PLR, and FC to serve as biomarkers of mucosal severity in UC.

\section{Statistical Analysis}

Student $t$-test was used to compare continuous data between patients with UC and healthy controls. The Mann-Whitney $U$ test was used to analyze the continuous variables. The receiver operating characteristic (ROC) curve analysis was performed to assess the performance of each biomarker for differentiating mucosal severity in UC. The sensitivity, specificity, and cutoff values were assessed using the ROC curve. A $P$-value $<0.05$ was considered to be statistically significant. The DeLong test was performed to compare the measures and the
$P$-value was adjusted by Bonferroni correction. All statistical analyses were performed using SPSS version 21.0 (SPSS Inc., Chicago, IL, USA) and R version 3.6.1 ('pRoc' and 'OptimalCutpoints' packages; R Foundation for Statistical Computing, Vienna, Austria).

\section{RESULTS}

\section{Study Population}

The electronic medical records of 144 subjects including 48 patients with UC and 96 healthy controls were reviewed during the study period. The mean age of the patients with UC was $38.9 \pm 14.8$ years. The male: female ratio was 1.18 (males 26 , females 22). The mean Mayo score was $6.8 \pm 2.3$. The clinical characteristics of the 48 patients with UC are summarized in Table 1.

\section{Comparisons of Serum Biomarkers between Patients with UC and Healthy Controls}

WBC, NLR, PLR, ESR, and CRP level was significantly higher in patients with UC versus healthy controls. NLR $(3.24 \pm 2.78$ vs. $1.52 \pm 0.61)$ and PLR $(187.01 \pm 136.94$ vs. $132.88 \pm 45.72)$ were considerably elevated in patients with UC versus healthy controls (Table 2). In patients with UC, ESR $(43.45 \pm 29.96$ $\mathrm{mm} / \mathrm{hr}$ vs. $18.85 \pm 15.81 \mathrm{~mm} / \mathrm{hr})$ and CRP $(0.79 \pm 1.43 \mathrm{mg} / \mathrm{dL}$ vs. $0.14 \pm 0.31 \mathrm{mg} / \mathrm{dL}$ ) were higher than the upper limit of the reference range (Table 2).

\section{Comparisons of Serum Biomarkers between Mild to Moderate UC and Severe UC}

NLR, PLR, ESR, CRP, and FC were significantly higher in patients with severe UC (group 2) versus mild to moderate UC (group 1). PLR was higher in group 2 versus group 1 (280.04 \pm 106.44 vs. $159.35 \pm 133.81, P<0.001)$. NLR also was higher in

Table 2. Comparisons of Serum Biomarkers between Patients with UC and Healthy Controls

\begin{tabular}{|c|c|c|c|c|}
\hline Variable & UC group $(n=48)$ & Control group $(n=96)$ & $P$-value & Reference \\
\hline WBC $(/ \mu \mathrm{L})$ & $7,750.00 \pm 2,932.21$ & $5,335.42 \pm 1,271.46$ & $<0.001$ & $4,000-10,000$ \\
\hline NLR & $3.24 \pm 2.78$ & $1.52 \pm 0.61$ & $<0.001$ & - \\
\hline PLR & $187.01 \pm 136.94$ & $132.88 \pm 45.72$ & $<0.001$ & - \\
\hline ESR $(\mathrm{mm} / \mathrm{hr})$ & $43.45 \pm 29.96$ & $18.85 \pm 15.81$ & $<0.001$ & $0-30$ \\
\hline $\mathrm{CRP}(\mathrm{mg} / \mathrm{dL})$ & $0.79 \pm 1.43$ & $0.14 \pm 0.31$ & $<0.001$ & $0.0-0.5$ \\
\hline
\end{tabular}

Values are presented as mean \pm standard deviation.

UC, ulcerative colitis; WBC, white blood cell; NLR, neutrophil to lymphocyte ratio; PLR, platelet to lymphocyte ratio; ESR, erythrocyte sedimentation rate; CRP, C-reactive protein. 
group 2 versus group $1(4.04 \pm 2.25$ vs. $3.00 \pm 2.91, P=0.034)$. FC was significantly increased in group 2 versus group $1(2,476.09 \pm$ $2,572.13 \mu \mathrm{g} / \mathrm{g}$ vs. $575.04 \pm 1,181.98 \mu \mathrm{g} / \mathrm{g}, P=0.002$ ) (Table 3 ).

\section{Comparison of the Diagnostic Accuracy for Predicting UC Using NLR and PLR}

ROC analysis was performed to determine the cutoff of NLR and PLR to predict UC. To differentiate patients with UC from healthy controls, WBC had the highest area under the curve (AUC) among NLR, PLR, ESR, and CRP. However, it had the lowest positive likelihood ratio as compared with the other parameters (sensitivity $72.9 \%$; specificity $74.0 \%$; positive likelihood ratio 2.800, 95\% confidence interval [CI], 1.917-4.089; AUC $0.793,95 \%$ CI 0.713-0.874). ROC analysis revealed a sensitivity of $54.2 \%$ and specificity of $90.6 \%$ when an NLR cutoff of 2.26 was used (positive likelihood ratio 5.778, 95\% CI 2.944-

Table 3. Comparisons of Serum Biomarkers According to Endoscopic Severity in UC Patients

\begin{tabular}{lcccc}
\hline Variable & $\begin{array}{c}\text { Group 1 }(\mathrm{n}=37) \\
\text { (mild to moderate) }\end{array}$ & $\begin{array}{c}\text { Group 2 }(\mathrm{n}=11) \\
\text { (severe) }\end{array}$ & P-value & \multicolumn{1}{c}{ Reference } \\
\hline WBC $(/ \mu \mathrm{L})$ & $7,635.14 \pm 2,917.88$ & $8,136.36 \pm 3,089.75$ & $0.508-10,000$ \\
NLR & $3.00 \pm 2.91$ & $4.04 \pm 2.25$ & 0.034 & - \\
PLR & $159.35 \pm 133.81$ & $280.04 \pm 106.44$ & $<0.001$ & - \\
ESR $(\mathrm{mm} / \mathrm{hr})$ & $37.75 \pm 29.06$ & $62.09 \pm 25.99$ & 0.007 & $0-30$ \\
CRP $(\mathrm{mg} / \mathrm{dL})$ & $0.45 \pm 0.97$ & $1.90 \pm 2.09$ & $<0.001$ & $0.0-0.5$ \\
FC $(\mu \mathrm{g} / \mathrm{g})$ & $575.04 \pm 1,181.98$ & $2,476.09 \pm 2,572.13$ & 0.002 & $0-100$ \\
\hline
\end{tabular}

Values are presented as mean \pm standard deviation.

UC, ulcerative colitis; WBC, white blood cell; NLR, neutrophil to lymphocyte ratio; PLR, platelet to lymphocyte ratio; ESR, erythrocyte sedimentation rate; CRP, C-reactive protein; FC, fecal calprotectin.
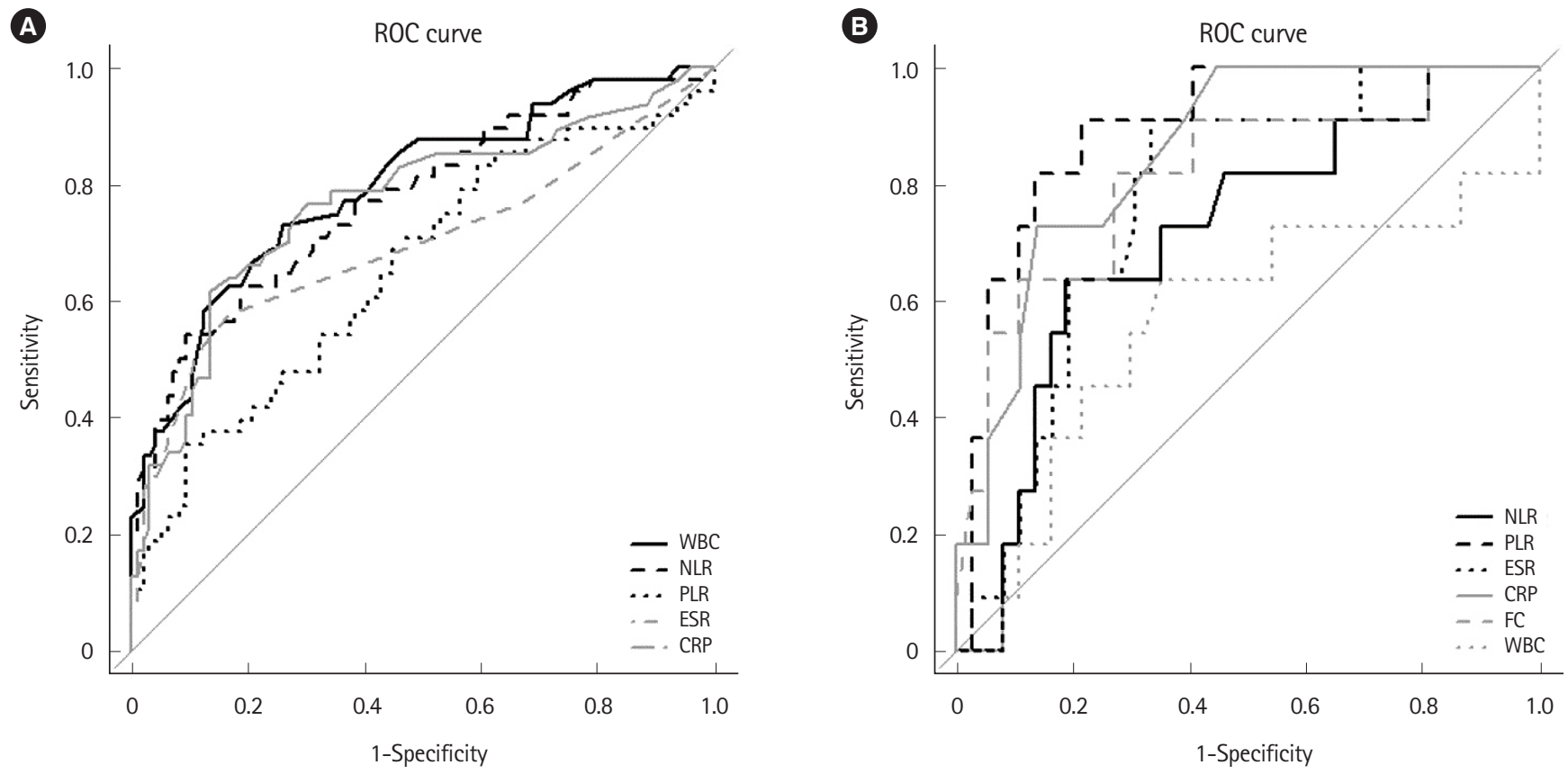

Fig. 2. ROC curve showing the diagnostic performance of $N L R, P L R$, and FC. (A) In the ROC curve, the optimal cutoff value for NLR and PLR for detecting UC was 2.26 (sensitivity 54.2\%; specificity 90.6\%; AUC 0.774, 95\% Cl 0.690-0.859) and 179.8 (sensitivity 35.4\%; specificity 90.6\%, AUC 0.654, 95\% Cl 0.556-0.753). (B) The optimal cutoff value for NLR, PLR, and FC for differentiating UC severity were 3.44 (sensitivity 63.6\%; specificity 81.1\%; AUC 0.714, 95\% Cl 0.539-0.888), 175.9 (sensitivity 90.9\%; specificity 78.4\%; AUC 0.897, 95\% Cl, 0.802-0.992), and $453 \mu \mathrm{g} / \mathrm{g}$ (sensitivity 81.8\%; specificity 73.0\%; AUC 0.813, 95\% Cl, 0.655-0.972), respectively. ROC, receiver operator characteristic; NLR, neutrophil to lymphocyte ratio; PLR, platelet to lymphocyte ratio; FC, fecal calprotectin; UC, ulcerative colitis; $A U C$, area under the curve; $\mathrm{Cl}$, confidence interval; WBC, white blood cell; ESR, erythrocyte sedimentation rate; CRP, C-reactive protein. 


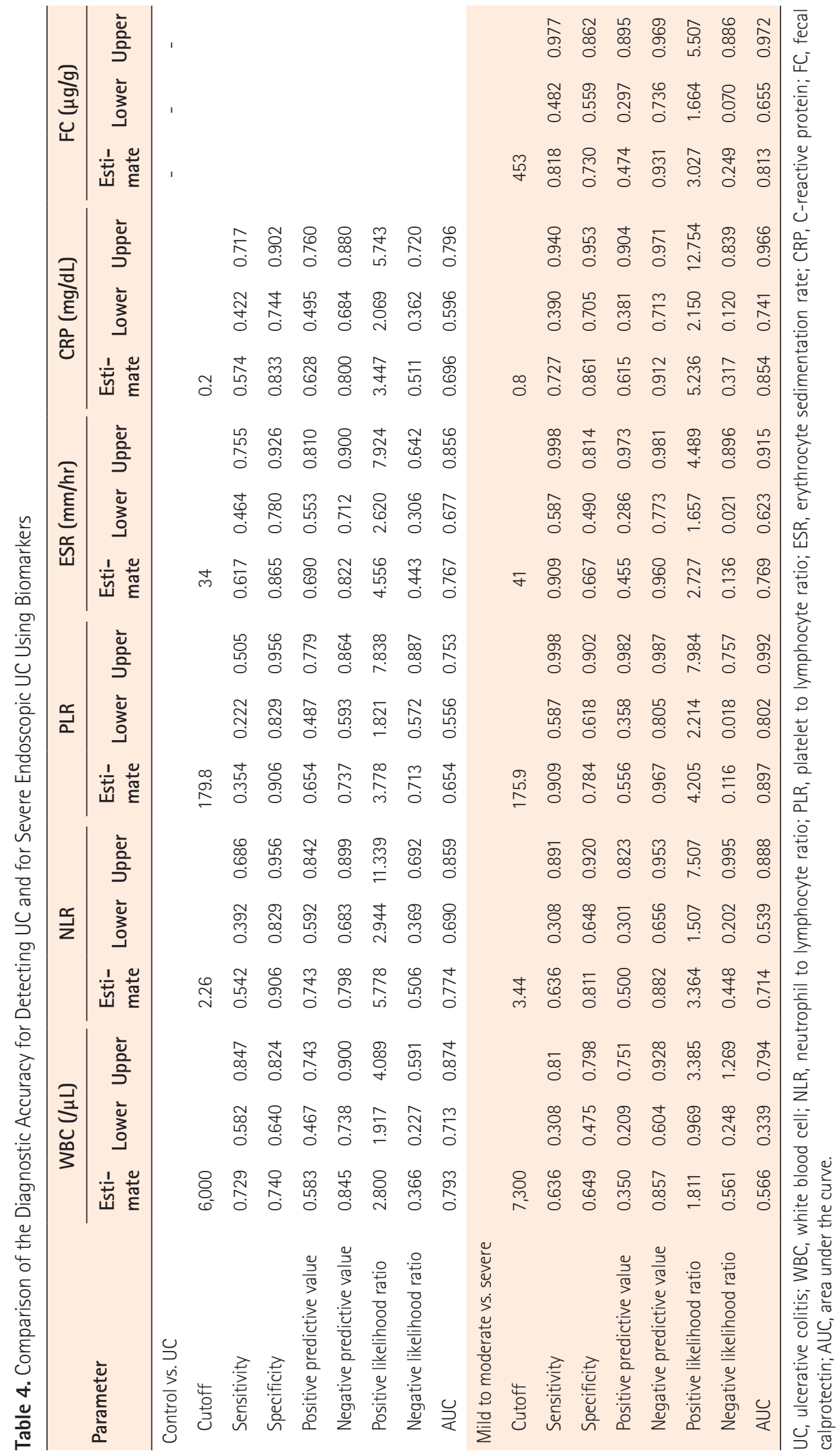


Table 5. Comparisons between Biomarkers on Healthy Control versus UC and Mild to Moderate versus Severe UC

\begin{tabular}{|c|c|c|c|c|c|}
\hline \multirow{2}{*}{\multicolumn{2}{|c|}{ Parameter }} & \multicolumn{2}{|c|}{ Control vs. UC } & \multicolumn{2}{|c|}{ Mild to moderate vs. severe } \\
\hline & & \multirow{2}{*}{$\frac{P \text {-value }}{0.690}$} & \multirow{2}{*}{$\begin{array}{c}\text { Adjusted } P \text {-value }^{\mathrm{b}} \\
1.000\end{array}$} & \multirow{2}{*}{$\frac{P \text {-value }}{\text { a }}$} & \multirow{2}{*}{$\begin{array}{c}\text { Adjusted } P \text {-value } \\
0.560\end{array}$} \\
\hline WBC & PLR & & & & \\
\hline & NLR & 0.036 & 0.363 & 0.005 & 0.071 \\
\hline & ESR & 0.712 & 1.000 & 0.143 & 1.000 \\
\hline & CRP & 0.131 & 1.000 & 0.046 & 0.693 \\
\hline & $\mathrm{FC}$ & - & - & 0.122 & 1.000 \\
\hline \multirow[t]{4}{*}{ PLR } & NLR & 0.006 & 0.059 & 0.017 & 0.165 \\
\hline & ESR & 0.962 & 1.000 & 0.680 & 1.000 \\
\hline & CRP & 0.190 & 1.000 & 0.214 & 1.000 \\
\hline & $\mathrm{FC}$ & - & - & 0.457 & 1.000 \\
\hline \multirow[t]{3}{*}{ NLR } & ESR & 0.061 & 0.612 & 0.045 & 0.449 \\
\hline & CRP & 0.511 & 1.000 & 0.490 & 1.000 \\
\hline & $\mathrm{FC}$ & - & - & 0.374 & 1.000 \\
\hline \multirow[t]{2}{*}{ ESR } & CRP & 0.181 & 1.000 & 0.260 & 1.000 \\
\hline & FC & - & - & 0.714 & 1.000 \\
\hline CRP & FC & - & - & 0.674 & 1.000 \\
\hline
\end{tabular}

${ }^{a} P$-value by DeLong test.

${ }^{b} P$-value adjusted by Bonferroni correction.

UC, ulcerative colitis; WBC, white blood cell; PLR, platelet to lymphocyte ratio; NLR, neutrophil to lymphocyte ratio; ESR, erythrocyte sedimentation rate; $C R P, C$-reactive protein; $F C$, fecal calprotectin.

11.339; AUC 0.774, 95\% CI 0.690-0.859). For identifying UC, the optimal cutoff of 179.8 for PLR had a sensitivity of $35.4 \%$ and a specificity of $90.6 \%$ (positive likelihood ratio $3.778,95 \%$ CI 1.821-7.838; AUC 0.654, 95\% CI 0.556-0.753) (Table 4, Fig. 2A). NLR had a significantly higher AUC versus PLR $(P=0.006)$ before Bonferroni correction, but the difference was no longer statistically significant after correction (Tables 4,5 ).

\section{Comparison of the Diagnostic Accuracy for Severe Endoscopic UC Using NLR, PLR, and FC}

The optimal cutoff for differentiating group 1 and 2 patients with UC was 3.44 (sensitivity $63.6 \%$; specificity $81.1 \%$; positive likelihood ratio 3.364, 95\% CI 1.507-7.507; AUC 0.714, 95\% CI 0.539-0.888) for NLR, 175.9 (sensitivity 90.9\%; specificity 78.4\%; positive likelihood ratio 4.205, 95\% CI 2.214-7.894; AUC 0.897, 95\% CI 0.802-0.992) for PLR, and $453 \mu \mathrm{g} / \mathrm{g}$ (sensitivity 81.8\%; specificity 73.0\%; positive likelihood ratio 3.027, 95\% CI 1.6645.507; AUC 0.813, 95\% CI 0.655-0.972) for FC (Table 4, Fig. 2B). PLR had the highest AUC among NLR, PLR, ESR, CRP, and FC and it had a higher positive likelihood ratio than NLR and FC. The AUC for PLR was significantly higher than NLR $(P=0.017)$ before Bonferroni correction but the difference was no longer statistically significant after the correction (Tables 4, 5).

\section{DISCUSSION}

In this study, we investigated the accuracy of NLR, PLR, and FC to diagnose UC and their ability to reflect disease activity. First, our results suggest that elevated NLR and PLR help differentiate patients with UC from healthy controls. Second, NLR, PLR, and FC indicated endoscopic activity. To the best of our knowledge, though previous studies have compared NLR and PLR with clinical indices, no study has fully examined the relationship between NLR, PLR, and FC with endoscopic activity in UC.

Mucosal healing, defined as the absence of ulcerations and erosions, is assessed by endoscopy and is the treatment goal in UC as it may prevent relapse and complications and minimize the need for hospitalization or surgery. ${ }^{14-16}$ Although endoscopy is a valuable tool to identify mucosal inflammation, it is invasive, inconvenient, and may be inappropriate in severe cases as it can cause major complications such as a perforation. ${ }^{17}$ Therefore, noninvasive biomarkers such as WBC, CRP, 
and ESR have been used to identify intestinal inflammation in patients with IBD, albeit with insufficient sensitivity. ${ }^{18,19}$

NLR and PLR can diagnose and predict the severity of IBD., ${ }^{2,20-22}$ Our study also suggests that elevated NLR and PLR help differentiate patients with UC from healthy controls. WBC, including neutrophils (that reflect systemic inflammation), contribute to the innate and adaptive immunity and these cells migrate to the inflamed tissues by releasing proinflammatory cytokines and chemokines. ${ }^{23}$ An elevated platelet count can contribute to the pathogenesis of mucosal inflammation by its proinflammatory properties such as the release and recruitment of inflammatory mediators and modulation of other inflammatory cells. ${ }^{24-26}$ In contrast, a reduced lymphocyte count in UC may result from mucosal infiltration. ${ }^{27}$ This results in an elevation of NLR and PLR, which was considerably elevated in patients with UC versus healthy controls in our study. A cutoff of 2.26 for NLR and 179.8 for PLR suggested UC. NLR was more significant than PLR for diagnosing UC. In previous studies, the optimal cutoff for NLR and PLR was 2.13-3.10 and 139, respectively which is similar to our results (2.26 for NLR and 179.8 for PLR). ${ }^{2,21,22}$ The cutoff for PLR was slightly higher than previously reported. The difference in PLR cutoff between our study and the previous study may influence the number of enrolled patients and the use of drugs such as azathioprine, steroids, anti-tumor necrosis factor (anti-TNF) and disease activity.

Our results demonstrated that NLR, PLR, and FC reflect intestinal mucosal conditions in UC. Recently, stool tests such as FC have been suggested as novel biomarkers. FC is a calcium- and zinc-binding protein that comprises $60 \%$ of the neutrophil-cytosolic protein. ${ }^{28} \mathrm{~A}$ high level of FC in IBD can be due to an increased neutrophil migration into the intestinal mucosa and an increased leukocyte turnover. ${ }^{29}$ However, FC requires stool sampling, ${ }^{21,30,31}$ and is relatively expensive as compared with NLR and PLR. In this study, PLR had the highest AUC among NLR, PLR, ESR, CRP, and FC. Although there were no significant differences, these results suggest that a high PLR was more meaningful to measure the severity of mucosal inflammation than FC. Interestingly, NLR is a more significant biomarker than PLR to differentiate patients with UC from healthy controls, but PLR was more significant in distinguishing severe UC from mild to moderate UC. This difference could be due to a comparison between different groups. Therefore, our study suggests that NLR and PLR should be considered together when evaluating and treating patients with UC.

This study has several limitations. First, it was a retrospec- tive, single-center study with a relatively small sample size. Second, there may be a selection bias as only patients with UC who underwent both endoscopy and FC testing were enrolled. This limits the strength of our conclusions. Third, FC data were not available for controls because this test is not routinely performed as part of a health examination. However, NLR and PLR, which were calculated from CBC, can be used routinely as a noninvasive and low-cost biomarker for identifying UC as per our results. Fourth, although CBC (especially absolute neutrophil counts) is affected by drugs such as azathioprine, steroids, and anti-TNF, we did not exclude all patients who took these drugs. However, the difference in neutrophil, lymphocyte, platelet, NLR, and PLR was not statistically significant between the patients on these drugs versus the patients on only 5 -aminosalicylic acid. This may be because we included a relatively small number of patients on these medications, and those with an abnormal CBC had previously adjusted the drug dose or changed the medication. Finally, we could not use blood and FC tests performed on the same day as the endoscopy for patients with UC. For more precise comparisons between FC or other biomarkers and endoscopic activity, patients must provide stool and blood samples on the day of endoscopy. However, in clinical practice, these tests are not usually performed on the same day. Therefore, the interval between FC and endoscopy may have contributed to the relatively low correlation between them as compared with previous studies. The results of our study should be interpreted in light of these limitations. To overcome these limitations, prospective studies, including larger cohorts are needed. Despite these limitations, our results suggest that an elevated PLR and NLR instead of FC could be used to indicate endoscopic activity and differentiate patients with UC from healthy controls in real practice.

In conclusion, both NLR and PLR can serve as biomarkers to differentiate patients with UC from healthy controls. These ratios may also reflect the condition of the intestinal mucosal, especially in patients with UC where colonoscopy is not possible.

\section{ADDITIONAL INFORMATION}

\section{Funding Source}

This work was supported by the Soonchunhyang University Research Fund. 


\section{Conflict of Interest}

Jeon SR is an editorial board member of the journal but did not involve in the peer reviewer selection, evaluation, or decision process of this article. No other potential conflicts of interest relevant to this article were reported.

\section{Author Contribution}

Conceptualization: Jeon SR. Methodology: Jeon SR. Formal analysis: Jeong Y, Jeon SR. Acquisition of data: Moon JR, Cho JH, Park JS, Park H, Lee KH, Ko BM. Visualization: Park S. Writing - original draft: Jeong Y. Writing - review and editing: Jeon SR, Kim HG, Lee TH, Jang JY, Lee JS, Kim JO. Approval of final manuscript: all authors.

\section{ORCID}

Jeong $\mathrm{Y}$

Jeon SR

Kim HG

Moon JR

Lee TH

Jang JY

Cho JH

Park JS

Park H

Lee $\mathrm{KH}$

Kim JO

Lee JS

Ko BM

Park S

\section{REFERENCES}

1. Singh P, Ananthakrishnan A, Ahuja V. Pivot to Asia: inflammatory bowel disease burden. Intest Res 2017;15:138-141.

2. Demir AK, Demirtas A, Kaya SU, et al. The relationship between the neutrophil-lymphocyte ratio and disease activity in patients with ulcerative colitis. Kaohsiung J Med Sci 2015;31: 585-590.

3. Cintolo M, Costantino G, Pallio S, Fries W. Mucosal healing in inflammatory bowel disease: maintain or de-escalate therapy. World J Gastrointest Pathophysiol 2016;7:1-16.

4. Menees SB, Powell C, Kurlander J, Goel A, Chey WD. A metaanalysis of the utility of C-reactive protein, erythrocyte sedimentation rate, fecal calprotectin, and fecal lactoferrin to exclude inflammatory bowel disease in adults with IBS. Am J Gastroenterol 2015;110:444-454.
5. Agassandian M, Shurin GV, Ma Y, Shurin MR. C-reactive protein and lung diseases. Int J Biochem Cell Biol 2014;53:77-88.

6. Emerging Risk Factors Collaboration, Kaptoge S, Di Angelantonio E, et al. C-reactive protein, fibrinogen, and cardiovascular disease prediction. N Engl J Med 2012;367:1310-1320.

7. Jang HW, Kim HS, Park SJ, et al. Accuracy of three different fecal calprotectin tests in the diagnosis of inflammatory bowel disease. Intest Res 2016;14:305-313.

8. Buderus S, Boone J, Lyerly D, Lentze MJ. Fecal lactoferrin: a new parameter to monitor infliximab therapy. Dig Dis Sci 2004; 49:1036-1039.

9. Sipponen T, Kärkkäinen P, Savilahti E, et al. Correlation of faecal calprotectin and lactoferrin with an endoscopic score for Crohn's disease and histological findings. Aliment Pharmacol Ther 2008;28:1221-1229.

10. Sipponen T, Savilahti E, Kolho KL, Nuutinen H, Turunen U, Färkkilä M. Crohn's disease activity assessed by fecal calprotectin and lactoferrin: correlation with Crohn's disease activity index and endoscopic findings. Inflamm Bowel Dis 2008;14: 40-46.

11. Akpinar MY, Ozin YO, Kaplan M, et al. Platelet-to-lymphocyte ratio and neutrophil-to-lymphocyte ratio predict mucosal disease severity in ulcerative colitis. J Med Biochem 2018;37:155162.

12. Silverberg MS, Satsangi J, Ahmad T, et al. Toward an integrated clinical, molecular and serological classification of inflammatory bowel disease: report of a Working Party of the 2005 Montreal World Congress of Gastroenterology. Can J Gastroenterol 2005;19 Suppl A:5A-36A.

13. Schroeder KW, Tremaine WJ, Ilstrup DM. Coated oral 5-aminosalicylic acid therapy for mildly to moderately active ulcerative colitis: a randomized study. N Engl J Med 1987;317:16251629.

14. Dave M, Loftus EV Jr. Mucosal healing in inflammatory bowel disease-a true paradigm of success? Gastroenterol Hepatol (N Y) 2012;8:29-38.

15. Park JJ, Yang SK, Ye BD, et al. Second Korean guidelines for the management of Crohn's disease. Intest Res 2017;15:38-67.

16. Choi CH, Moon W, Kim YS, et al. Second Korean guidelines for the management of ulcerative colitis. Intest Res 2017;15:737.

17. Mukewar S, Costedio M, Wu X, et al. Severe adverse outcomes of endoscopic perforations in patients with and without IBD. Inflamm Bowel Dis 2014;20:2056-2066.

18. Magro F, Gionchetti P, Eliakim R, et al. Third European evidencebased consensus on diagnosis and management of ulcerative 
colitis. Part 1: definitions, diagnosis, extra-intestinal manifestations, pregnancy, cancer surveillance, surgery, and ileo-anal pouch disorders. J Crohns Colitis 2017;11:649-670.

19. Gomollón F, Dignass A, Annese V, et al. 3rd European evidencebased consensus on the diagnosis and management of Crohn's disease 2016. Part 1: diagnosis and medical management. J Crohns Colitis 2017;11:3-25.

20. Torun S, Tunc BD, Suvak B, et al. Assessment of neutrophillymphocyte ratio in ulcerative colitis: a promising marker in predicting disease severity. Clin Res Hepatol Gastroenterol 2012;36:491-497.

21. Gao SQ, Huang LD, Dai RJ, Chen DD, Hu WJ, Shan YF. Neutrophil-lymphocyte ratio: a controversial marker in predicting Crohn's disease severity. Int J Clin Exp Pathol 2015;8:1477914785.

22. Celikbilek M, Dogan S, Ozbakır O, et al. Neutrophil-lymphocyte ratio as a predictor of disease severity in ulcerative colitis. J Clin Lab Anal 2013;27:72-76.

23. Segal AW. How neutrophils kill microbes. Annu Rev Immunol 2005;23:197-223.

24. Collins CE, Rampton DS. Platelet dysfunction: a new dimension in inflammatory bowel disease. Gut 1995;36:5-8.
25. Kapsoritakis AN, Potamianos SP, Sfiridaki AI, et al. Elevated thrombopoietin serum levels in patients with inflammatory bowel disease. Am J Gastroenterol 2000;95:3478-3481.

26. Collins CE, Cahill MR, Newland AC, Rampton DS. Platelets circulate in an activated state in inflammatory bowel disease. Gastroenterology 1994;106:840-845.

27. Selby WS, Jewell DP. T lymphocyte subsets in inflammatory bowel disease: peripheral blood. Gut 1983;24:99-105.

28. Konikoff MR, Denson LA. Role of fecal calprotectin as a biomarker of intestinal inflammation in inflammatory bowel disease. Inflamm Bowel Dis 2006;12:524-534.

29. Erbayrak M, Turkay C, Eraslan E, Cetinkaya H, Kasapoglu B, Bektas M. The role of fecal calprotectin in investigating inflammatory bowel diseases. Clinics (Sao Paulo) 2009;64:421-425.

30. Langhorst J, Elsenbruch S, Koelzer J, Rueffer A, Michalsen A, Dobos GJ. Noninvasive markers in the assessment of intestinal inflammation in inflammatory bowel diseases: performance of fecal lactoferrin, calprotectin, and PMN-elastase, CRP, and clinical indices. Am J Gastroenterol 2008;103:162-169.

31. Sutherland AD, Gearry RB, Frizelle FA. Review of fecal biomarkers in inflammatory bowel disease. Dis Colon Rectum 2008;51:1283-1291. 